\title{
Multiple effects of ellagic acid on human colorectal carcinoma cells identified by gene expression profile analysis
}

\author{
JINLU ZHAO ${ }^{1,2^{*}},{\text { GUODONG } \mathrm{LI}^{1,2^{*}} \text {, WANLAN BO }}^{3}$, YUHUI ZHOU ${ }^{1,2}$, \\ SHUWEI DANG ${ }^{1,2}$, JIUFENG WEI $^{1,2}$, XINGLONG LI $^{1,2}$ and MING LIU ${ }^{1,2}$ \\ ${ }^{1}$ Department of General Surgery, ${ }^{2}$ Bio-Bank of Department of General Surgery, and ${ }^{3}$ Department of Gastroenterology, \\ The Fourth Affiliated Hospital of Harbin Medical University, Harbin, Heilongjiang 150001, P.R. China
}

Received August 17, 2016; Accepted November 25, 2016

DOI: $10.3892 /$ ijo. 2017.3843

\begin{abstract}
Colorectal carcinoma (CRC) is the third most commonly diagnosed cancer in the world. Phytochemicals have become a research hotspot in recent years as cancer prevention and treatment agents due to their low toxicity and limited side-effects. Ellagic acid (EA), a natural phenolic constituent, displays various biological activities, including anticancer effects. However, the detailed anticancer mechanisms of EA remain unclear. In the present study, we found that EA inhibited the growth of HCT-116 colon cancer cells. Moreover, we identified differentially expressed genes (DEGs) by microarray profiling of HCT-116 cells treated with EA. A total of 857 DEGs (363 upregulated and 494 downregulated) were identified with a $>1.5$-fold change in expression after treatment with EA for $72 \mathrm{~h}$. Gene Ontology (GO) and Kyoto Encyclopedia of Genes and Genomes (KEGG) pathway analysis showed that a large number of cellular functions were modified by EA including proliferation, apoptosis, cell cycle and angiogenesis. Interaction network analysis using DEGs provided details of their interactions and predicted the key target pathways of EA. To verify the result of cDNA microarray, 10 selected DEGs related to proliferation, apoptosis or cell cycle were further confirmed by real-time RT-PCR. Based on microarray data, we identified several crucial functions of EA. These results provide important new data for EA in anti-CRC research.
\end{abstract}

Correspondence to: Professor Ming Liu, Department of General Surgery, The Fourth Affiliated Hospital of Harbin Medical University, No. 37 Yiyuan Street, Harbin, Heilongjiang 150001, P.R. China

E-mail: mliu35@aliyun.com

${ }^{*}$ Contributed equally

Key words: ellagic acid, colorectal carcinoma, microarray profiling, differentially expressed genes, Gene Ontology analysis, Kyoto Encyclopedia of Genes and Genomes pathway analysis, multi-target

\section{Introduction}

Colorectal carcinoma (CRC) is the third most common cancer and the second leading cause of tumor related deaths in the world (1). Given the global distribution of morbidity from CRC, it is apparent that the environmental factors play a significant role in its etiology. The connection between the diet and the incidence of CRC is now well-established (2). Although surgery, radiotherapy, chemotherapy and targeted therapies for CRC are improving, the existing treatment methods cannot completely control the high incidence or low survival rates of CRC. Chemoprevention has emerged recently as one of the most practical and effective tools to reduce the risk of cancer (3). Bioactive substances from plants are a source for novel antitumor drugs, and polyphenolic compounds, in particular, have been the focus of increasing interest due to their strong anticancer activity $(4,5)$.

Ellegic acid (EA; 2,3,7,8-tetrahydroxy-chromeno [5,4, 3-cde] chromene-5,10-dione; International Union of Pure and Applied Chemistry) is a polyphenolic compound abundant in woody plants, berries, grapes and nuts (6). EA has been found to exert both preventive and therapeutic effects against numerous human types of cancer, including colon, skin, prostate, breast and esophageal cancer (7-10). A number of studies have investigated the mechanisms of EA in the inhibition of carcinogenesis. Recent research has demonstrated that it suppresses cancer cell proliferation and migration by downregulation of VEGF-induced angiogenesis, VEGF-2 tyrosine kinase activity, and downstream MAPK and PI3K/Akt signaling pathways (10). EA also inhibits the invasive potential of tumors through its effects on the activity of proteases, such as collagenase/gelatinase and collagenase IV (11). In addition, EA can reduce the cancer cell viability by increasing the caspase-3 activity, downregulating Bcl-2 and decreasing the activity of telomerase (12). Although these studies focus on the mechanisms of EA in various signaling pathways, they failed to comprehensively encompass all of its biological activities. Moreover, the molecular effects of EA in inhibition of human CRC cells remain to be thoroughly elucidated.

In the present study, we used a high-throughput GeneChip containing $>20,000$ known genes to identify multiple targets affected by EA in human colon adenocarcinoma HCT-116 cells. This cDNA microarray method detects changes in gene 
expression profiles, providing evidence for the effects of anticancer agents on cancer cells (13). The GeneChip results were further confirmed by the real-time RT-PCR. Multiple different functions of EA were revealed in human CRC cells, providing vital data that will be of significant value to researchers.

\section{Materials and methods}

EA and cell lines. EA was purchased from Sigma Chemical Co. (St. Louis, MO, USA). A stock solution of EA was prepared in dimethyl sulfoxide (DMSO) and filter sterilized before use. The human CRC cell line, HCT-116, was purchased from the Cell Bank of Shanghai Institute of Biological Sciences, Chinese Academy of Sciences (Shanghai, China). HCT-116 cells were cultured in Dulbecco's modified Eagle's medium (DMEM) supplemented with $10 \%$ fetal bovine serum (FBS) and $1 \%$ penicillin streptomycin solution in an atmosphere of $95 \%$ air and $5 \% \mathrm{CO}_{2}$ in a $37^{\circ} \mathrm{C}$ humidified incubator.

Cell proliferation assay. HCT-116 cells were seeded in 96-well plates at a density designed to reach $\sim 80 \%$ confluence. Cells were allowed to adhere and $24 \mathrm{~h}$ later were treated with EA at $0,25,50,75,100$ and $125 \mu \mathrm{M}$. After 24,48 or $72 \mathrm{~h}$ of treatment, $10 \mu \mathrm{l}$ of MTT was added to $100 \mu \mathrm{l}$ culture medium per well. After $4 \mathrm{~h}$ of incubation at $37^{\circ} \mathrm{C}$, the medium was removed and $150 \mu \mathrm{l}$ DMSO was added. The absorbance was measured at a wavelength of $490 \mathrm{~nm}$ in a plate reader (Bio-Rad Laboratories, Hercules, CA, USA). Cell viability was calculated according to: OD sample/OD control $\mathrm{x} 100 \%$. The assay was performed in triplicate.

RNA extraction. After $72 \mathrm{~h}$ of EA treatment, total RNA was extracted using TRIzol reagent (Life Technologies, Carlsbad, CA, USA) and the RNeasy kit (Qiagen, Hilden, Germany) according to the manufacturer's instructions. The purity and integrity of the ribosomal RNA was checked.

Transcriptome microarray assay. Affymetrix Human Transcriptome Array 2.0 arrays (HTA 2.0) were hybridized according to the Affymetrix recommendations, using the Ambion WT protocol and Affymetrix Labeling and Hybridization kits. GeneChips were scanned using Affymetrix ${ }^{\circledR}$ GeneChip Command Console (AGCC). Data were manipulated using the Expression Console software (Affymetrix) at the Institut Curie Microarray Core Facility.

Differential gene expression analysis. A random variance model t-test was used to filter differentially expressed genes (DEGs) between the the control and the experimental groups. We selected DEGs according to a P-value threshold corrected using the false discovery rate (FDR) method, and a corrected $\mathrm{P}<0.05$ was considered statistically significant (14-16).

Gene Ontology $(\mathrm{GO})$ analysis. To analyze the main functions of the DEGs identified by microarray analysis, we used the Gene Ontology (the key functional classification from NCBI), which organizes genes into hierarchical categories and reveals regulatory networks based on the molecular functions and biological processes $(17,18)$. Due to the hierarchical relationships between the GO terms, the number of genes in each term varies widely and can be between one and several hundred genes. For a given marginal frequency (i.e. a constant total number of genes in a GO term), the probability of a gene being affected by a treatment obeys a hypergeometric distribution and P-values can be calculated by Fisher's exact test. Essentially, the P-value of a two-tailed Fisher's exact test solves the cumulative hypergeometric distribution values.

Specifically, two-sided Fisher's exact and $\chi^{2}$ tests were used to analyze GO categories, and the FDR was used to correct P-values (19). Scores to assess the enrichment of GOs were calculated using the following formula:

$$
\operatorname{Re}=\left(\frac{n_{f}}{n}\right) /\left(\frac{N_{f}}{N}\right)
$$

where ' $n_{f}$ ' is the number of DEGs in a GO, ' $n$ ' is the number of genes in a GO, ' $N_{f}$ ' is the total number of DEGs, and ' $N$ ' is the total number of genes in the annotation system (20).

Pathway analysis. Pathway analysis is used to determine the significant pathways in which DEGs participate. Since entire biochemical processes, including metabolism, signal transduction and the cell cycle, are described as 'pathways', a single pathway usually contains hundreds of genes. Fisher's exact and $\chi^{2}$ tests were used to detect regulatory pathways differing significantly according to the Kyoto Encyclopedia of Genes and Genomes (KEGG), BioCarta and Reactome, and significance thresholds were defined by FDR-corrected P-values (21-23).

Interaction networks of DEGs. Gene network analysis using the KEGG database is used to construct systems of interactions and overcome the limitations of determining interactions among genes in a single pathway. Genes with discordant records of upregulation or downregulation of mRNA expression in the database were excluded from the analysis and were recorded as having a negative association by default, which was not taken into account in multi-group differentiation analysis. The intersection was taken as the result. Therefore, gene signaling network analysis was able to determine the upstream and downstream molecules for proteins throughout the KEGG pathway database.

An interaction network was constructed based on the DEG data. Network maps were constructed using Java, which allows users to build and analyze molecular networks. Analysis was based on the KEGG interaction database. Networks were stored and presented as graphs, where nodes are mainly genes (proteins and complexes) and edges represent types of relationships between nodes, such as activation or phosphorylation.

The degree is defined as the number of links of one node with all other nodes. For a gene in a network, the number of source genes connecting to a gene is called its indegree, while the number of target genes connected to by a gene is its outdegree. The properties of genes are described by measures of betweenness centrality, which reflects the importance of a node in modulation of other nodes (24-28).

Real-time reverse transcription-PCR analysis. Changes in the expression of ten selected genes responding to EA were further assessed by quantitative RT-PCR. A total of $2 \mu \mathrm{g}$ of RNA from independent experiments was used to perform reverse 
Table I. The primers used for real-time RT-PCR analysis.

\begin{tabular}{|c|c|}
\hline Gene & Primer sequence \\
\hline$\beta$-actin & $\begin{array}{l}\text { CTCACCATGGATGATGATATCGC } \\
\text { AGGAATCCTTCTGACCCATGC }\end{array}$ \\
\hline IL8 & $\begin{array}{l}\text { CACCGGAAGGAACCATCTCA } \\
\text { TGGCAAAACTGCACCTTCACA }\end{array}$ \\
\hline$J U N$ & $\begin{array}{l}\text { CСАACTCATGCTAACGCAGC } \\
\text { СТСТCCGTCGCAACTTGTCA }\end{array}$ \\
\hline$C C N B 1$ & $\begin{array}{l}\text { TGGTGAATGGACACCAACTCT } \\
\text { TAGCATGCTTCGATGTGGCA }\end{array}$ \\
\hline IRS1 & $\begin{array}{l}\text { ACATCACAGCAGAATGAAGACCT } \\
\text { TGGATGCATCGTACCATCTACTG }\end{array}$ \\
\hline$P L K 1$ & $\begin{array}{l}\text { CAAGTACGGCCTTGGGTATCA } \\
\text { GTGCCGTCACGCTCTATGTA }\end{array}$ \\
\hline$C D C 20$ & $\begin{array}{l}\text { ATTCCCAGGTGTGCTCCATC } \\
\text { GCCATGGTTGGGTACTTCCA }\end{array}$ \\
\hline SMC3 & $\begin{array}{l}\text { CAGACAACCGGTTACCAATCG } \\
\text { AGCGCTTTCAAGGAGGTTCA }\end{array}$ \\
\hline$B C L-2$ & $\begin{array}{l}\text { AGATTGATGGGATCGTTGCCT } \\
\text { AGTCTACTTCCTCTGTGATGTTGT }\end{array}$ \\
\hline$B A D$ & $\begin{array}{l}\text { TCСTTTAAGAAGGGACTTCCTCG } \\
\text { CCAAGTTCCGATCCCACCAG }\end{array}$ \\
\hline$C-M Y C$ & $\begin{array}{l}\text { ССТАСССТСТCAACGACAGC } \\
\text { TTCСТССТCAGAGTCGCTGC }\end{array}$ \\
\hline
\end{tabular}

transcription using PrimeScript ${ }^{\mathrm{TM}}$ RT reagent kit (Takara Bio, Tokyo, Japan). Real-time quantitative PCR of transcribed cDNA was performed with SYBR Premix Ex Taq ${ }^{\text {TM }}$ II (Takara Bio). Primers were designed using Primer 5 software and synthesized by Comate Bioscience, Co., Ltd. (Changchun, China). The primers are listed in Table I. Real-time RT-PCR reactions were then performed in a total of $25 \mu \mathrm{l}$ of reaction mixture using the ABI Prism 7500HT sequence detection system (Applied Biosystems, Foster City, CA, USA). Data were analyzed using the comparative $\mathrm{Ct}$ method, and the expression levels of target genes were normalized to the levels of $\beta$-actin expression in each sample.

Statistical analysis. All data were presented as the mean \pm SD of six independent experiments. Two-tailed Student's t-test and one-way analysis of variance (ANOVA) were used to analyze significant differences. $\mathrm{P}<0.05$ was considered to indicate a statistically significant result.

\section{Results}

The effects of EA on HCT-116 cell proliferation. MTT assays were used to assess the anti-proliferative effects of EA and to select the appropriate concentration of EA and treatment duration for the microarray assay. As shown in Fig. 1, HCT-116 cells were incubated with different concentrations of EA for

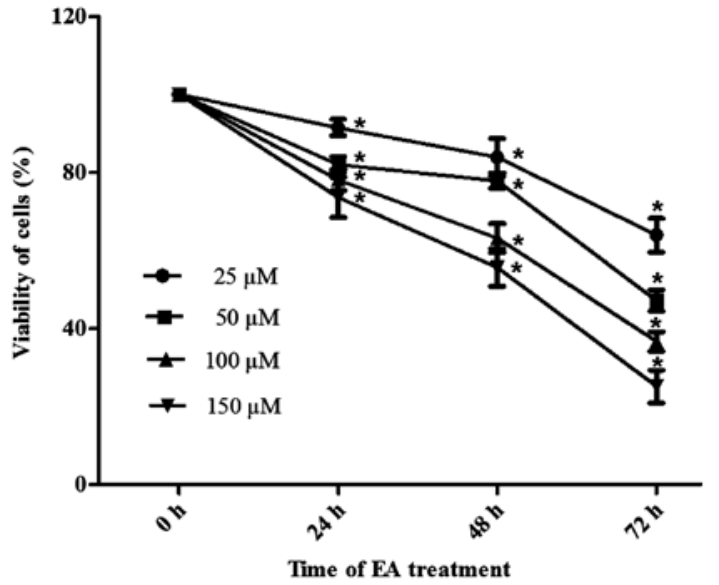

Figure 1. Effect of EA on the growth of HCT-116 cells. HCT-116 cells were exposed to increasing dosages of EA for 24,48 or $72 \mathrm{~h}$. Cell viability is expressed as a percentage of the control group (DMSO). Data are presented as means $\pm \mathrm{SD}, \mathrm{n}=6$ per group. ${ }^{*} \mathrm{P}<0.05$ compared to the vehicle control.

24, 48 or 72 h. EA exhibited anti-proliferative effects, which were both time- and dose-dependent.

Screening for differentially expressed genes. After treatment of HCT-116 cells with $100 \mu \mathrm{M}$ EA for $72 \mathrm{~h}$, microarray analysis indicated that a total of 857 genes had expression levels changed by $\geq 1.5$-fold (494 upregulated and 363 downregulated) (Fig. 2; $\mathrm{P}<0.05)$. The top $10 \mathrm{DEGs}$ are presented in Table II.

GO analysis. Comprehensive GO analysis of DEGs was performed to determine the main functions of EA in cancer inhibition. Thirty-eight GO terms significantly associated with differential gene expression after EA treatment (Fig. 3; $\mathrm{P}<0.05)$ were classified into key functional categories. The main GO categories identified included regulation of the apoptotic process, cell division, signal transduction, negative regulation of cell proliferation, gene expression, transcription and cell cycle arrest. Larger enrichment values indicate that the functions were affected more strongly by EA treatment.

Pathway analysis of DEGs. KEGG pathway analysis was performed to further investigate the key pathways associated with DEGs. Significant pathway categories $(\mathrm{P}<0.05)$ for the 857 DEGs linked to EA are presented in Fig. 4. Larger -Lg (P-values) indicate that the function was more strongly regulated in response to treatment with EA. Treatment with EA clearly affected 24 significant pathways, including the p53 signaling pathway, metabolic pathways, the PI3K-Akt signaling pathway, the MAPK signaling pathway, the TGF- $\beta$ signaling pathway and the cell cycle and transcriptional misregulation in cancer.

Interaction networks of DEG. Interaction-relationship networks of DEGs were constructed and are shown in Figs. 5 and 6. DEGs of interest were closely connected, and the majority of them were located in the center of the network. Betweenness centrality indicated the intermediary ability of each gene, and a larger value indicates a greater ability to 


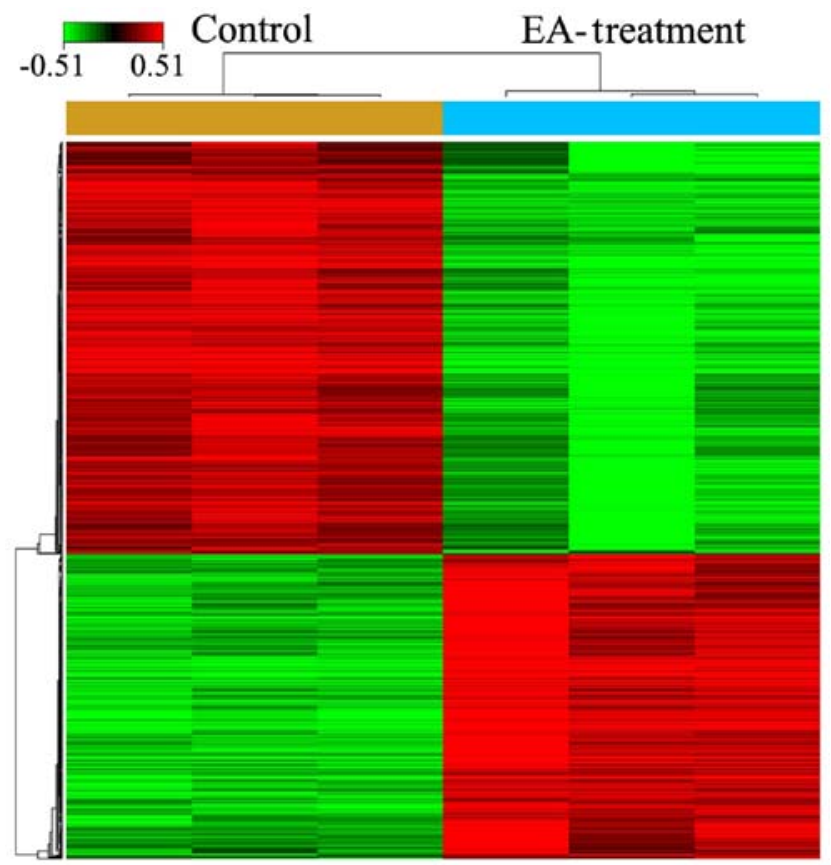

Figure 2. Heat map of DEGs with fold change expression $>1.5$. HCT-116 cells were treated with $100 \mu \mathrm{M}$ EA for $72 \mathrm{~h}(\mathrm{n}=3)$ or treated with vehicle control $(\mathrm{n}=3)$ Rows represent genes and columns represent samples. Red blocks represent high and green blocks low expression relative to comparison cells.

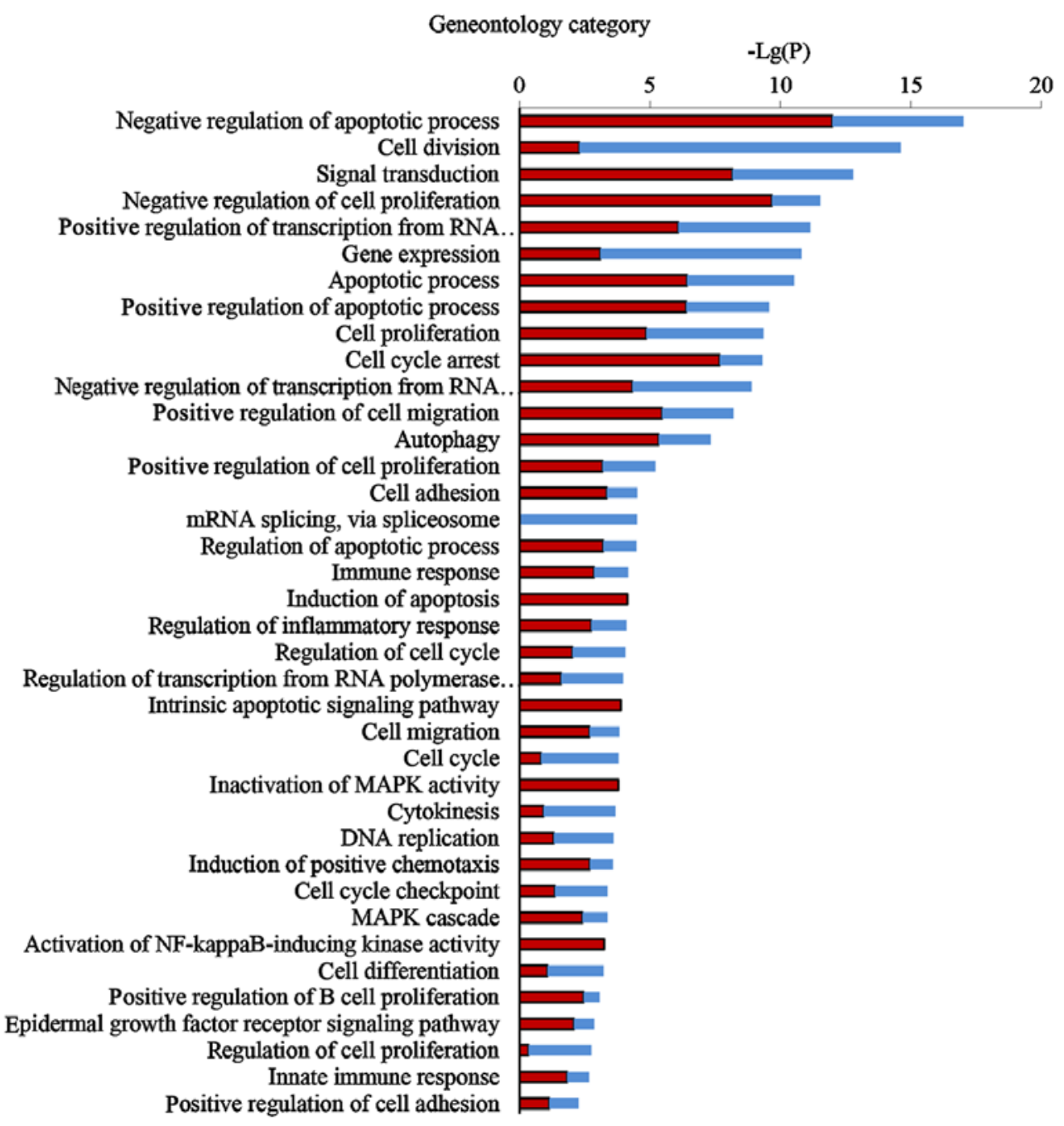

Figure 3. Enrichment of GO categories. Blue columns indicate the percentage of upregulated genes and red columns indicate the percentage of downregulated genes in each category. 


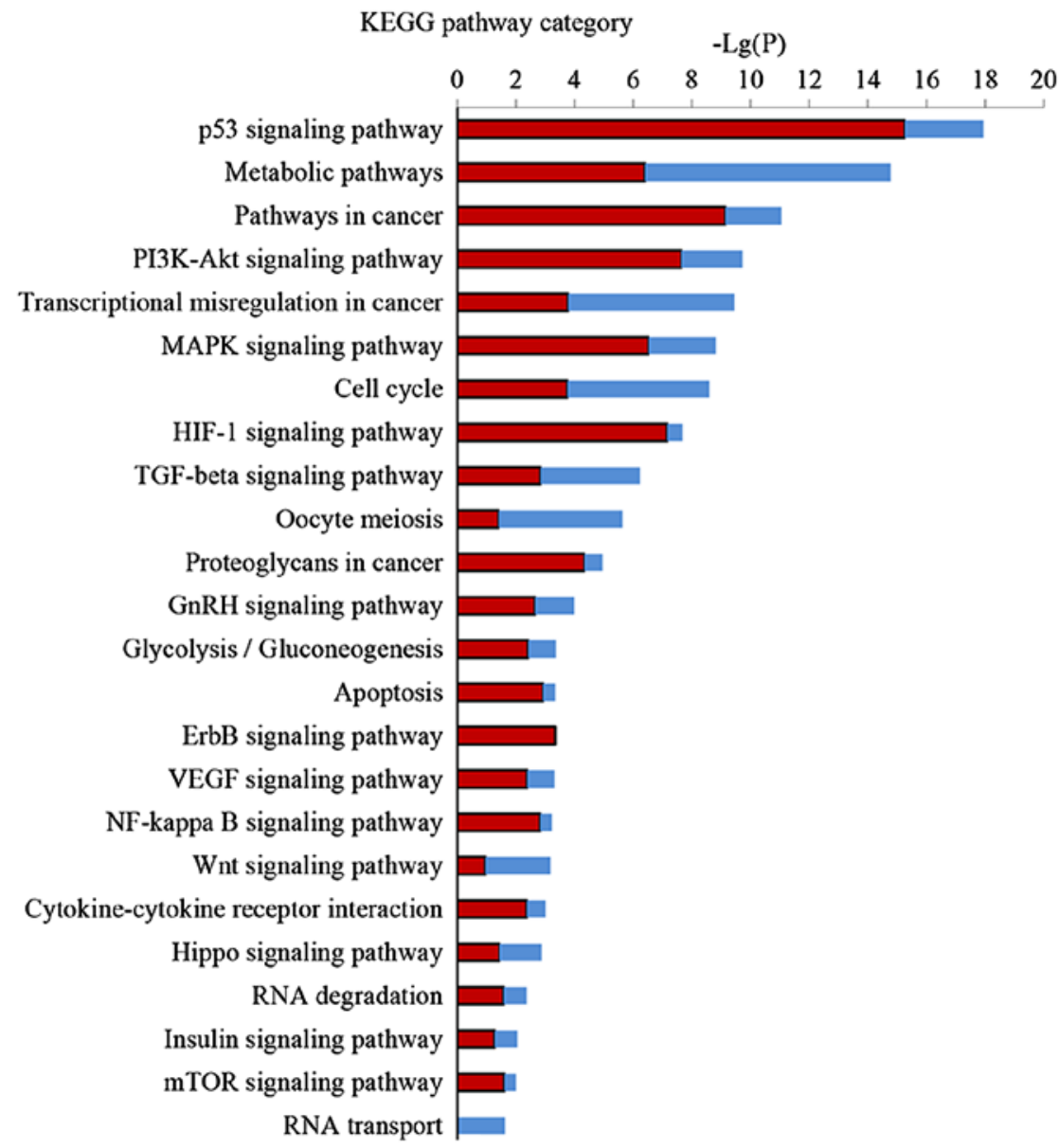

Figure 4. KEGG pathway analysis of HCT-116 cells after EA treatment. Blue columns indicate the percentage of upregulated genes and red columns indicate the percentage of downregulated genes in each pathway.

Table II. Top 10 regulated genes in EA-treatment cells compared with the control cells in the HCT-116 cells.

\begin{tabular}{cllcc}
\hline Rank & Gene symbol & Gene feature & Fold change & P-value \\
\hline 1 & CPA4 & Upregulated & 7.393308 & $5.10 \mathrm{E}-05$ \\
2 & ABCC2 & Downregulated & -6.93316 & $5.60 \mathrm{E}-05$ \\
3 & CENPE & Downregulated & -5.35232 & $6.10 \mathrm{E}-05$ \\
4 & CENPF & Downregulated & -4.48105 & $8.00 \mathrm{E}-05$ \\
5 & BHLHE40 & Upregulated & 4.231785 & $7.00 \mathrm{E}-05$ \\
6 & HIST1H2BM & Downregulated & -4.22724 & $9.50 \mathrm{E}-05$ \\
7 & KRTAP2-3 & Upregulated & 4.124822 & 0.000148 \\
8 & ID3 & Downregulated & -3.97283 & 0.000163 \\
9 & GDF15 & Upregulated & 3.684665 & 0.000265 \\
10 & HIST1H2AB & Downregulated & -3.65796 & 0.00025 \\
\hline
\end{tabular}

regulate genes. Fig. 6 shows the interaction network of DEGs related to proliferation, apoptosis, angiogenesis and cell cycle. These DEGs include a number of important functional genes such as PRKACB, IL8, JUN, CDC20 and CCNB1.

Real-time reverse transcription-PCR analysis. The next step was to confirm the changes of genes in microarray analysis by the real-time RT-PCR analysis. We selected 10 genes ( 3 upregulated, 6 downregulated and 1 no change by microarray data) which were related to proliferation, apoptosis or cell cycle. Nine out of the ten gene expression levels were verified by the real-time RT-PCR. This represents a success rate of $90 \%$ in microarray analysis. However, when the relative ratio of change for genes in microarray exceeded 2 , the success rate became $100 \%$ ( 7 out of 7 ). The results are shown in Table III. 


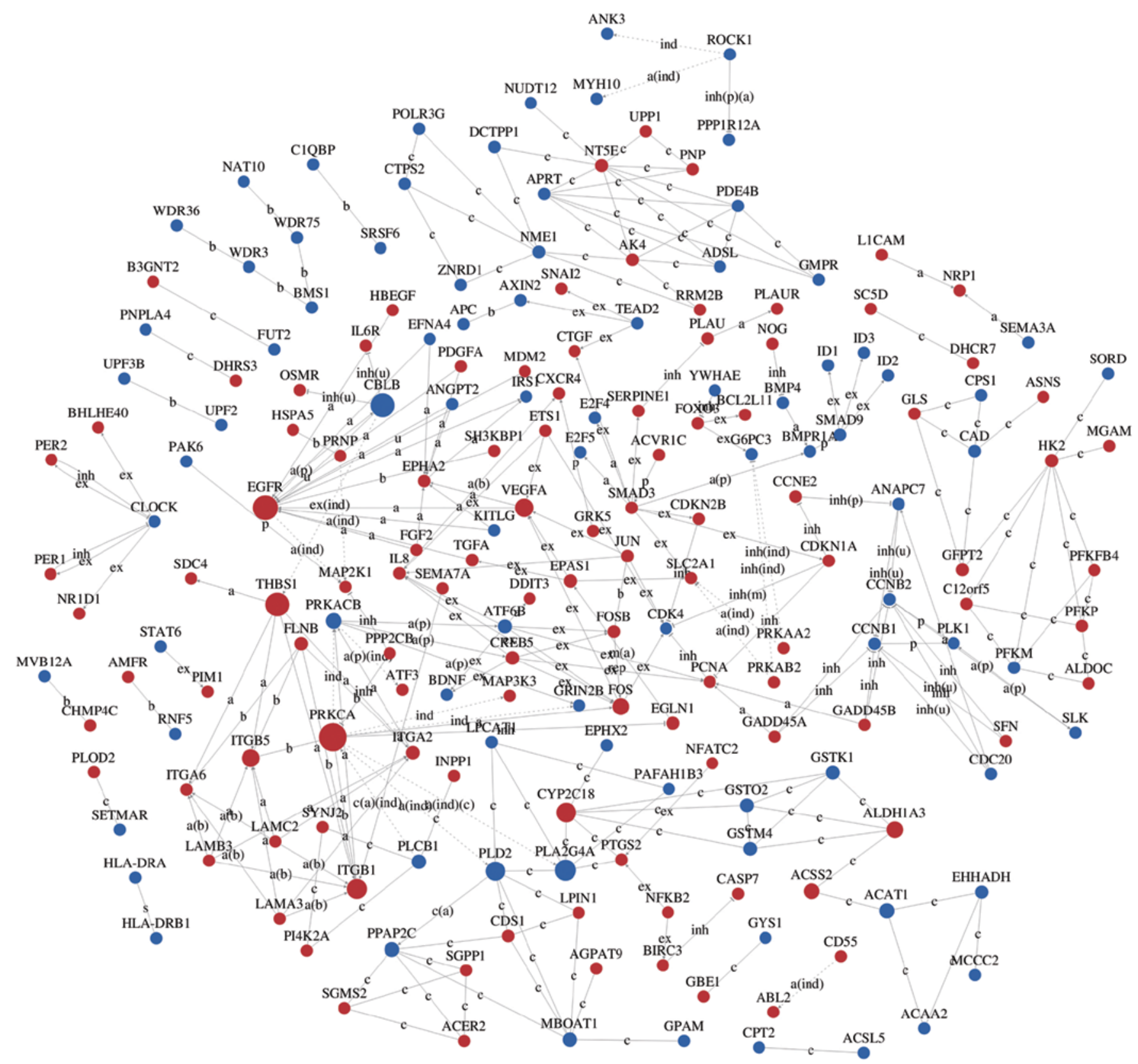

Figure 5. Gene-gene interaction network of HCT-116 cells after EA treatment. The area of circles is proportional to the value of betweenness centrality. Line segments indicate gene-gene interactions. Red indicates upregulated genes and blue indicates downregulated genes.

\section{Discussion}

Chemoprevention is emerging as an effective method for inhibiting cancer cells. Many plant polyphenols exhibit substantial inhibitory activity against the growth of colon cancer cells in vitro and against colon carcinogenesis in animal models (29). EA is regarded as one of the most promising and practical chemopreventive agents against various cancers (30). A previous study showed that the cytotoxicity and anti-proliferative activity of EA against cancer cells was detected at a concentration range that did not affect normal cell viability (31). In vitro and in vivo experiments have revealed that EA elicits substantial inhibitory effects against $\mathrm{CRC}$, which suggests that edible EA may be of value in treatment or prevention of CRC (32-34). However, the molecular mechanisms at the protein and transcriptional levels involved in the cellular response to EA are not yet completely understood. Therefore, it is important to reveal the targets and molecular mechanisms of EA induced inhibition of CRC cell growth. To this end, we used microarray profiling, which has provided remarkable insights in many areas of modern medical research (35). The colon adenocarcinoma cell HCT-116 is widely investigated as a reliable model to check their anti-proliferative properties for various drugs $(36,37)$. The growth inhibitory effects of EA on colon cancer cells have been previously reported at concentrations $\geq 100 \mu \mathrm{M}$ in vitro studies (36). The present study showed that $\mathrm{IC}_{50}$ of EA on HCT-116 cells was $90.20 \mu \mathrm{M}$ (data not shown). Thus, we chose $100 \mu \mathrm{M}$ as the treatment concentration. In addition, based on literature, $72 \mathrm{~h}$ after the treatment is the time-point 


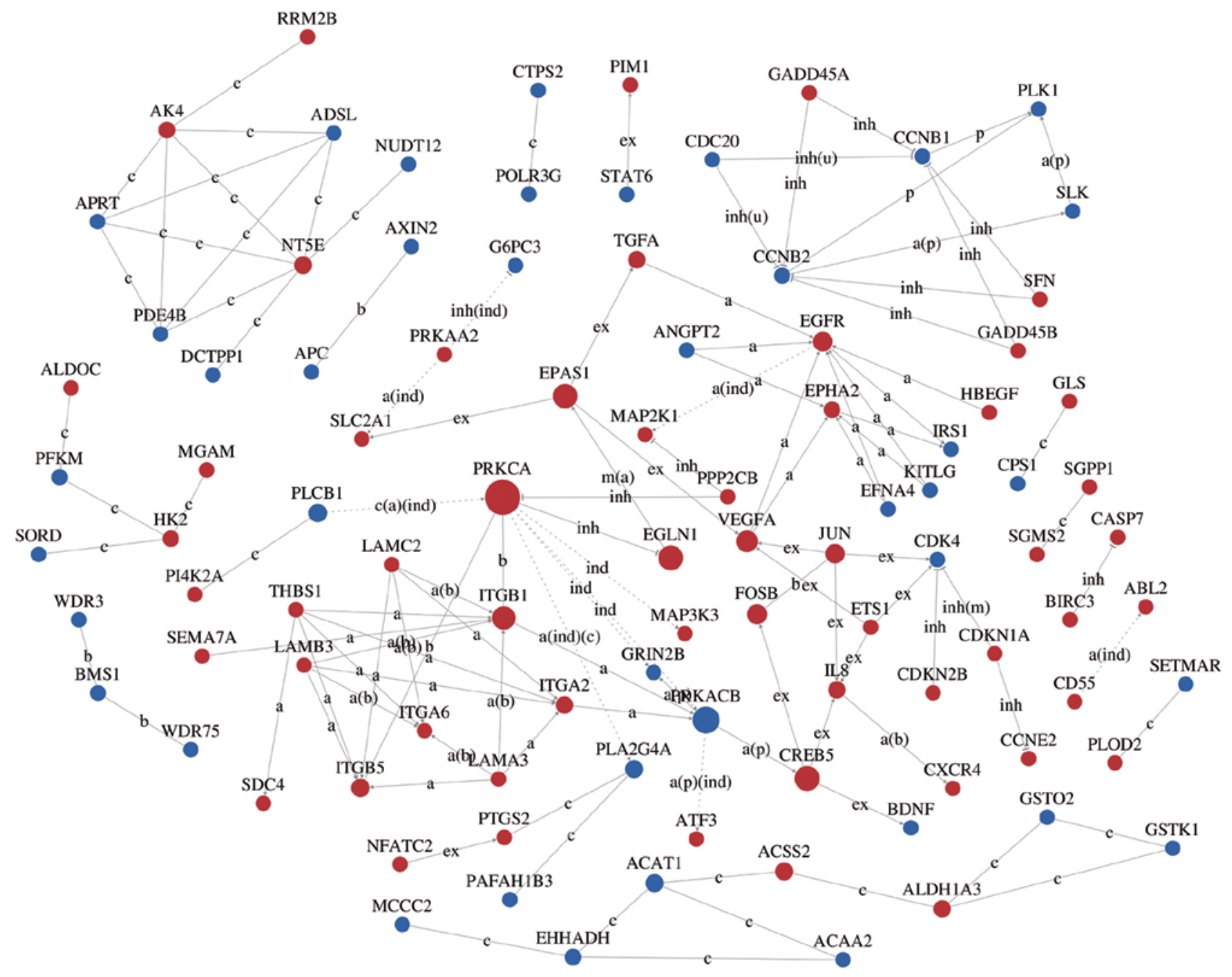

Figure 6. Regulation of proliferation, apoptosis and the cell cycle gene-gene interaction networks of HCT-116 cells after EA treatment.

often used for microarray experiment of antitumor drugs, including the EA, by evidence of significant changes of gene expression and morphology $(35,38)$. Preliminary screening for DEGs identified 857 genes (494 upregulated and 363 downregulated) in HCT-116 cells after $72 \mathrm{~h}$ of exposure to $\mathrm{EA}$, which are the colon adenocarcinoma cells widely investigated as a reliable model to check their anti-proliferative properties for various drugs. Among the top 10 DEGs in HCT-116 (Table II), Carboxypeptidase 4 (CPA4) is a zincdependent metallocarboxypeptidase on chromosome $7 \mathrm{q} 32$ in a region linked to prostate cancer aggressiveness. CPA4 is involved in the histone hyperacetylation pathway and may affect the growth and regulation of prostate epithelial cells (39). Centrosome-associated protein E (CENPE), a kinesin-like motor protein that accumulates in the G2 phase of the cell cycle, selectively leads to proliferation inhibition of basal-like breast cancer cell lines when inhibited. A study suggested that CENPE may be an effective therapeutic target for patients with triple-negative/basal breast cancer (40). At present, the relationships between the top 10 DEGs and the other common DEGs in the KEGG pathway database are rarely reported. Thus, they are not emerging from the results of the DEG interaction networks, making these genes important targets for our future research.
GO category analysis (Fig. 3) is becoming a standard procedure following many high throughput experimental studies, and it suggests novel hypothesis for follow-up works (41). Many key targets in CRC development and progression were identified as significantly regulated by EA. According to the P-values of each GO category, we found that the three most important functions regulated by EA were apoptotic process, cell proliferation and cell cycle arrest.

KEGG pathway analysis is the significant analysis of pathways in which these DEGs participate. Our results demonstrated 24 regulated pathways by EA, most of which are related to the apoptotic process, cell proliferation, or cell cycle arrest. EA can activate the PI3K/Akt pathway, which modulates Bcl-2 family proteins leading to an induction of apoptosis (42). EA also arrests the cell cycle of Caco-2 cells at the S- and G2/Mphases through regulation of key genes in the MAPK pathways including EGFR, KRAS, MYC, FOS and CCNB1 (42).

According to the results described above, we constructed gene-gene interaction networks (Figs. 5 and 6) to investigate the relationships among the groups of genes. The results indicate that some established key genes play important roles in the mediation of the effects of EA treatment, including IL8, ETS1, JUN and CCNB1, which are involved in cell cycle arrest (43). IL8 is a chemokine which acts on a common receptor, CXCR3, 


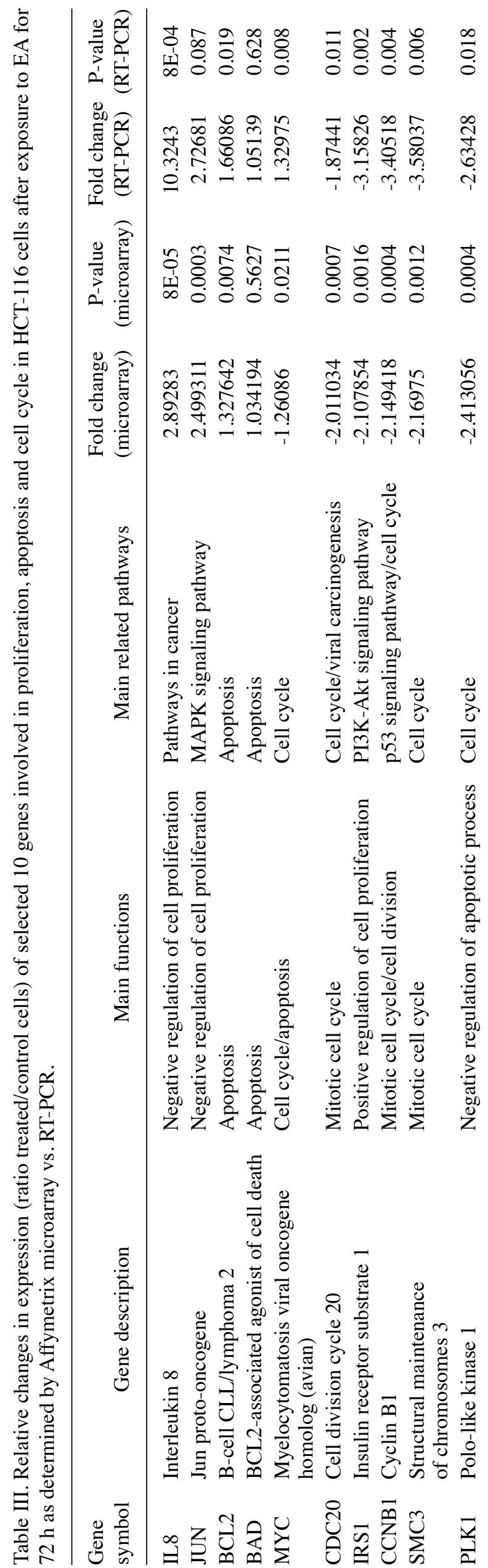

to increase cell migration (44). EA also reduced the levels of CCNB1 protein, which is involved in the control of the G2/M transition and mitosis in CRC cells $(42,45)$. Moreover, EA can inhibit cell proliferation in bladder cancer by downregulation of c-Jun, a subunit of activation protein 1 (46). However, since signaling pathways are interactive and complex, modulation of a single target is not always effective in cancer prevention and multi-targeted therapy is expected to improve treatment effectiveness. Further detailed analyses demonstrate that EA suppression of colon cancer HCT-116 cells is through simultaneous regulation of the expression of functional cancer target genes, including PRKACB, CCNB1, CDC20, JUN, MEF2C and IL8. Most of them are interacting and involved in the apoptotic process, cell proliferation or cell cycle arrest. Protein kinase $\mathrm{C}$ (PKC) is critical to cell proliferation, and the anti-carcinogenic action of EA has been confirmed to downregulate PKC (47). EA also inhibits the expression of markers of angiogenesis, including IL-8, VEGF and VEGFR, in mouse xenografts of the human pancreatic cancer cell line, PANC-1 (48). Moreover, the relative expression changes (ratio treated/control) of some functional genes were confirmed by RT-PCR (Table III). It is highly likely that these are the most important key targets of EA in HCT-116 cells.

These results showed that EA may play important roles in inhibiting CRC by regulating multiple targets and modulating key signaling pathways and fundamental cell processes. The results of the microarray analysis also implicate the immune response, DNA replication, and metabolism in responding to EA. A recent study found that EA induced cancer cell death by blocking energy metabolism (49). EA also significantly reduced the proliferation of human osteogenic sarcoma (HOS) cells by degrading chromosomal DNA (50). These altered functions will be the subject of our future research.

In conclusion, this study provided preliminary evidence of the antitumor effects of EA treatment on CRC cells. Microarray profiling demonstrated multiple effects of EA and provided a number of avenues for further research. Based on the results of microarray, further studies are needed to validate the multiple functions of EA and provide evidence to support its application in prevention and therapies for human CRC.

\section{Acknowledgements}

The present study was supported by the National Natural Science Foundation of China (nos. 81372612 and 81302059), the Foundation of Heilongjiang Educational Committee (no. 12541300) and the Study Abroad Returnees Science Foundation of Heilongjiang (no. LC2013C35).

\section{References}

1. World Cancer Research Fund International: Worldwide data. $<$ http://www.wcrf.Org/int/cancer-facts-figures/worldwide-data>, 2012.

2. Reddy BS and Cohen LA: Diet and colon cancer: evidence from human and animal model studies. CRC Press, Boca Raton, FL, pp47-65, 1986.

3. Cragg GM and Newman DJ: Plants as a source of anti-cancer agents. J Ethnopharmacol 100: 72-79, 2005.

4. Potter JD: Cancer prevention: Epidemiology and experiment. Cancer Lett 114: 7-9, 1997.

5. Potter JD and Steinmetz K: Vegetables, fruit and phytoestrogens as preventive agents. IARC Sci Publ 139: 61-90, 1996. 
6. Girish C and Pradhan SC: Drug development for liver diseases: Focus on picroliv, ellagic acid and curcumin. Fundam Clin Pharmacol 22: 623-632, 2008.

7. Heber D: Multitargeted therapy of cancer by ellagitannins. Cancer Lett 269: 262-268, 2008.

8. Zhang T, Chen HS, Wang LF, Bai MH, Wang YC, Jiang XF and Liu M: Ellagic acid exerts anti-proliferation effects via modulation of Tgf- $\beta / \mathrm{Smad} 3$ signaling in MCF-7 breast cancer cells. Asian Pac J Cancer Prev 15: 273-276, 2014.

9. Dai Z, Nair V, Khan M and Ciolino HP: Pomegranate extract inhibits the proliferation and viability of MMTV-Wnt-1 mouse mammary cancer stem cells in vitro. Oncol Rep 24: 1087-1091, 2010.

10. Wang N, Wang ZY, Mo SL, Loo TY, Wang DM, Luo HB, Yang DP, Chen YL, Shen JG and Chen JP: Ellagic acid, a phenolic compound, exerts anti-angiogenesis effects via VEGFR-2 signaling pathway in breast cancer. Breast Cancer Res Treat 134: 943-955, 2012

11. Pitchakarn P, Chewonarin T, Ogawa K, Suzuki S, Asamoto M, Takahashi S, Shirai T and Limtrakul P: Ellagic acid inhibits migration and invasion by prostate cancer cell lines. Asian Pac J Cancer Prev 14: 2859-2863, 2013.

12. Huang ST, Wang CY, Yang RC, Chu CJ, Wu HT and Pang JH: Phyllanthus urinaria increases apoptosis and reduces telomerase activity in human nasopharyngeal carcinoma cells. Forsch Komplement Med 16: 34-40, 2009.

13. Chen HS, Bai MH, Zhang T, Li GD and Liu M: Ellagic acid induces cell cycle arrest and apoptosis through TGF- $\beta / \mathrm{Smad} 3$ signaling pathway in human breast cancer MCF-7 cells. Int J Oncol 46: 1730-1738, 2015.

14. Wright GW and Simon RM: A random variance model for detection of differential gene expression in small microarray experiments. Bioinformatics 19: 2448-2455, 2003.

15. Yang H, Crawford N, Lukes L, Finney R, Lancaster $M$ and Hunter KW: Metastasis predictive signature profiles pre-exist in normal tissues. Clin Exp Metastasis 22: 593-603, 2005.

16. Clarke R, Ressom HW, Wang A, Xuan J, Liu MC, Gehan EA and Wang Y: The properties of high-dimensional data spaces: Implications for exploring gene and protein expression data. Nat Rev Cancer 8: 37-49, 2008

17. Gene Ontology Consortium: The Gene Ontology (GO) project in 2006. Nucleic Acids Res 34: D322-D326, 2006.

18. Ashburner M, Ball CA, Blake JA, Botstein D, Butler H, Cherry JM, Davis AP, Dolinski K, Dwight SS, Eppig JT, et al; Gene ontology: tool for the unification of biology. The Gene Ontology Consortium. Nat Genet 25: 25-29, 2000.

19. Dupuy D, Bertin N, Hidalgo CA, Venkatesan K, Tu D, Lee D, Rosenberg J, Svrzikapa N, Blanc A, Carnec A, et al: Genomescale analysis of in vivo spatiotemporal promoter activity in Caenorhabditis elegans. Nat Biotechnol 25: 663-668, 2007.

20. Schlitt T, Palin K, Rung J, Dietmann S, Lappe M, Ukkonen E and Brazma A: From gene networks to gene function. Genome Res 13: 2568-2576, 2003

21. Kanehisa M, Goto S, Kawashima S, Okuno Y and Hattori M: The KEGG resource for deciphering the genome. Nucleic Acids Res 32: D277-D280, 2004.

22. Yi M, Horton JD, Cohen JC, Hobbs HH and Stephens RM: WholePathwayScope: A comprehensive pathway-based analysis tool for high-throughput data. BMC Bioinformatics 7: 30, 2006.

23. Draghici S, Khatri P, Tarca AL, Amin K, Done A, Voichita C, Georgescu C and Romero R: A systems biology approach for pathway level analysis. Genome Res 17: 1537-1545, 2007.

24. Jansen R, Greenbaum D and Gerstein M: Relating whole-genome expression data with protein-protein interactions. Genome Res 12: 37-46, 2002

25. Li C and $\mathrm{Li} \mathrm{H}$ : Network-constrained regularization and variable selection for analysis of genomic data. Bioinformatics 24 1175-1182, 2008

26. Wei $\mathrm{Z}$ and Li H: A Markov random field model for network-based analysis of genomic data. Bioinformatics 23: 1537-1544, 2007.

27. Zhang JD and Wiemann S: KEGGgraph: A graph approach to KEGG PATHWAY in R and bioconductor. Bioinformatics 25 1470-1471, 2009.

28. Spirin V and Mirny LA: Protein complexes and functional modules in molecular networks. Proc Natl Acad Sci USA 100: 12123-12128, 2003

29. Rudolf E, Andelová H and Cervinka M: Polyphenolic compounds in chemoprevention of colon cancer - targets and signaling pathways. Anticancer Agents Med Chem 7: 559-575, 2007.
30. Whitley AC, Stoner GD, Darby MV and Walle T: Intestinal epithelial cell accumulation of the cancer preventive polyphenol ellagic acid - extensive binding to protein and DNA. Biochem Pharmacol 66: 907-915, 2003.

31. Losso JN, Bansode RR, Trappey A II, Bawadi HA and Truax R: In vitro anti-proliferative activities of ellagic acid. J Nutr Biochem 15: 672-678, 2004.

32. Cho H, Jung H, Lee H, Yi HC, Kwak HK and Hwang KT: Chemopreventive activity of ellagitannins and their derivatives from black raspberry seeds on HT-29 colon cancer cells. Food Funct 6: 1675-1683, 2015 .

33. Mertens-Talcott SU, Lee JH, Percival SS and Talcott ST: Induction of cell death in Caco-2 human colon carcinoma cells by ellagic acid rich fractions from muscadine grapes (Vitis rotundifolia). J Agric Food Chem 54: 5336-5343, 2006.

34. Umesalma S and Sudhandiran G: Differential inhibitory effects of the polyphenol ellagic acid on inflammatory mediators NF-kappaB, iNOS, COX-2, TNF-alpha, and IL-6 in 1,2-dimethylhydrazine-induced rat colon carcinogenesis. Basic Clin Pharmacol Toxicol 107: 650-655, 2010.

35. Kong X, Ding X and Yang Q: Identification of multi-target effects of Huaier aqueous extract via microarray profiling in triple-negative breast cancer cells. Int J Oncol 46: 2047-2056, 2015.

36. Seeram NP, Adams LS, Henning SM, Niu Y, Zhang Y, Nair MG and Heber D: In vitro antiproliferative, apoptotic and antioxidant activities of punicalagin, ellagic acid and a total pomegranate tannin extract are enhanced in combination with other polyphenols as found in pomegranate juice. J Nutr Biochem 16: 360-367, 2005.

37. Kang K, Oh SH, Yun JH, Jho EH, Kang JH, Batsuren D, Tunsag J, Park KH, Kim M and Nho CW: A novel topoisomerase inhibitor, daurinol, suppresses growth of HCT116 cells with low hematological toxicity compared to etoposide. Neoplasia 13: 1043-1057, 2011.

38. González-Sarrías A, Espín JC, Tomás-Barberán FA and GarcíaConesa MT: Gene expression, cell cycle arrest and MAPK signalling regulation in Caco-2 cells exposed to ellagic acid and its metabolites, urolithins. Mol Nutr Food Res 53: 686-698, 2009

39. Ross PL, Cheng I, Liu X, Cicek MS, Carroll PR, Casey G and Witte JS: Carboxypeptidase 4 gene variants and early-onset intermediate-to-high risk prostate cancer. BMC Cancer 9: 69, 2009.

40. Kung PP, Martinez R, Zhu Z, Zager M, Blasina A, Rymer I, Hallin J, Xu M, Carroll C, Chionis J, et al: Chemogenetic evaluation of the mitotic kinesin CENP-E reveals a critical role in triple-negative breast cancer. Mol Cancer Ther 13: 2104-2115, 2014.

41. Ihmels J, Friedlander G, Bergmann S, Sarig O, Ziv Y and Barkai N: Revealing modular organization in the yeast transcriptional network. Nat Genet 31: 370-377, 2002.

42. Umesalma S, Nagendraprabhu P and Sudhandiran G: Ellagic acid inhibits proliferation and induced apoptosis via the Akt signaling pathway in HCT-15 colon adenocarcinoma cells. Mol Cell Biochem 399: 303-313, 2015.

43. Viallard JF, Lacombe F, Belloc F, Pellegrin JL and Reiffers J: Molecular mechanisms controlling the cell cycle: Fundamental aspects and implications for oncology. Cancer Radiother 5: 109-129, 2001 (In French).

44. Billottet C, Quemener C and Bikfalvi A: CXCR3, a double-edged sword in tumor progression and angiogenesis. Biochim Biophys Acta 1836: 287-295, 2013

45. Larrosa M, Tomás-Barberán FA and Espín JC: The dietary hydrolysable tannin punicalagin releases ellagic acid that induces apoptosis in human colon adenocarcinoma Caco- 2 cells by using the mitochondrial pathway. J Nutr Biochem 17: 611-625, 2006.

46. Qiu Z, Zhou B, Jin L, Yu H, Liu L, Liu Y, Qin C, Xie S and Zhu F: In vitro antioxidant and antiproliferative effects of ellagic acid and its colonic metabolite, urolithins, on human bladder cancer T24 cells. Food Chem Toxicol 59: 428-437, 2013.

47. Mishra S and Vinayak M: Ellagic acid checks lymphoma promotion via regulation of PKC signaling pathway. Mol Biol Rep 40: 1417-1428, 2013.

48. Zhao M, Tang SN, Marsh JL, Shankar S and Srivastava RK: Ellagic acid inhibits human pancreatic cancer growth in Balb c nude mice. Cancer Lett 337: 210-217, 2013.

49. Mishra S and Vinayak M: Ellagic acid induces novel and atypical PKC isoforms and promotes caspase-3 dependent apoptosis by blocking energy metabolism. Nutr Cancer 66: 675-681, 2014

50. Han DH, Lee MJ and Kim JH: Antioxidant and apoptosisinducing activities of ellagic acid. Anticancer Res 26 (5A): 3601-3606, 2006 